\title{
XXVII. Auflösung einiger Aufgaben der stereographischen Projection.
}

\author{
Von \\ E. von Fedorow in St. Petersburg.
}

(Mil 7 Textfiguren.)

Ich erlaube mir hier auf einige Aufgaben der stereographischen Projection und ibre Auflösungen aufmerksam zu machen, deren ich mich mehrfach bei meiner Untersuchung der optischen Verbältnisse der Plagioklase in bequemer Weise bediente.

Die erste Gruppe derselben steht in engem Verbältnisse zu der bekannten Aufgabe, die Kreisbögen von sehr grossem Durchmesser zu ziehen. Die Aufgaben dieser Art bildeten den Gegenstand einer speciellen Abhandlung von Websk y*). Dieser Gelehrte hat aber eine sehr grosse Genauigkeit in Aussicht gehabt. Andererseits löst er die Aufgaben durch Rechnung und nicht durch Construction.

Bei meiner Untersuchung stand aber nicht die Genauigkeit, sondern die rasche und bequeme Ausführung im Vordergrunde. Deshalb war es nicht unzweckmässig, sich der folgenden angenäherten Methode zu bedienen.

Dieser liegt folgendes, den Mathematikern sehr gut bekanntes Princip zu Grunde.

Ziehen wir eine Curve $A B$ in BeFig. 1.

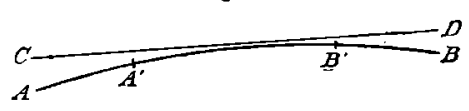
tracht (Fig. 1). Ein sehr kleiner Bogen $A^{\prime} B^{\prime}$ derselben lässt sich als ein Kreisbogen auffassen ; der Fehler, der dabei begangen wird, wird um so unbeträchtlicher, je kleiner der Bogen $A^{\prime} B^{\prime}$ ist. Der Kreisbogen, welcher dieselbe Tangente $C D$ mit der Curve und denselben Krummungsradius in dem Beruhrungspunkte hat, ist der Gurve in

*) Mittheilungen aus den Sitzungsber. d. k. pr. Akad. d. Wiss. zu Berlin 1886, Hft.1 (Ueber Construction flacber Zonenbögen). Ref. in dieser Zeitschr. 14, 77. 
solchem Grade angenähert, dass er mil ihr die Berührung zweit er Ordnung hat *).

Mit Hülfe dieses Princips wird folgende Auflösung unmittelbar verständlich.

Fig. 2.

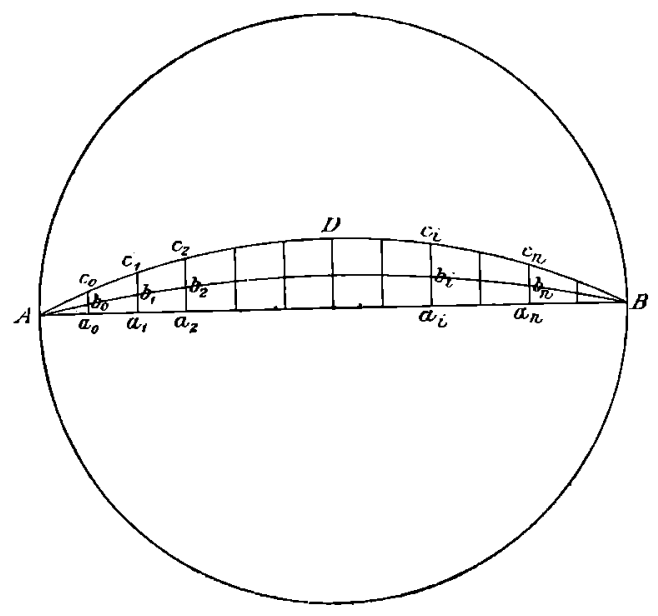

1. Aufgabe. Einen sehr flachen Kreisbogen durch die Enden $A$ und $B$ eines Diameters und durch den Punkt $b_{i}$ zu ziehen ${ }^{* *}$ ) (Fig. 2).

Wir ziehen zuerst einen beliebigen, aber möglichst flachen, durch die Punkte $A$ und $B$ hindurehgehenden Kreisbogen $A D B$, und dann eine Reihe zu $A B$ senkrechter Geraden $a_{0} b_{0}, a_{1} b_{1} \ldots a_{n} b_{n}$. An einer von dieser Geraden $a_{i} b_{i}$ finden wir das Verhälıniss $\frac{c_{i} a_{i}}{b_{i} a_{i}}$, und auf allen anderen

Geraden ergeben sich die Punkte $b_{0}, b_{1} \ldots b_{n}$, welche der Proportionalität $\frac{c_{0} a_{0}}{b_{0} a_{0}}=\frac{c_{1} a_{1}}{b_{1} a_{1}}=\ldots=\frac{c_{n} a_{n}}{b_{n} a_{n}}$ genügen.

Der durch die Punkte $A, b_{0}, b_{1} \ldots b_{i} \ldots b_{n}, B$ gehende, sehr flache Ellipsenbogen lässt sich mit genügender Strenge als ein Kreisbogen anseben.

Fig. 3.

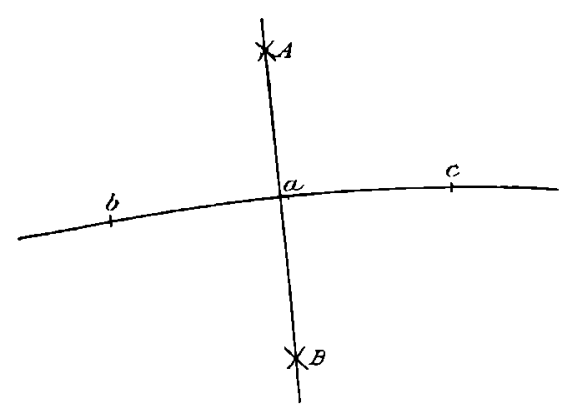

Die Construction lässt sich mit Hülfe des Proportionalzirkels sehr bequem und rasch vollziehen.

Ich will den so construirten Bogen einen genäherten Kreisbogen nennen.

$\mathrm{Z}$ weite Aufgabe. Auf einen genäherten Kreisbogen in einem gegebenen Punkte a die Normale zu ziehen (Fig. 3).

Infolge des Umstandes, dass der

*) Der bekannten Theorie der Berührung der Curven zufolge, welche wir Lagrang e verdanken.

**) Herr G. Wulff, mein hocbgeehrter College, hat mich mündlich mit seiner schönen Melhode bekannt gemacht, welcbe hoffentlich bald von ihm publicirt wird. 
genäherte Kreishogen dem wirklichen Kreisbogen sehr nahe steht, ist folgende, allgemein bekannte Construction anzuwenden :

Auf dem Bogen nehmen wir die gleichen Theile $a c=$ $a b$ und zieben dann aus den Punkten $b$ und $c$ als Centren die sich in den Punkten $A$ und $B$ schneidenden Kreisbögen von einem und demselben Durchmesser. Die Gerade $A B$ ist dic gesuchte Normale.

Dritte Aufgabe. Den Winkel zwischen zwei genäherten Zonenkreisen zu finden (Fig. 4).

Es seien $A B$ und $C D$ zwei gegebene genäherte Kreisbögen, $E$ ihr Durchschnittspunkt.

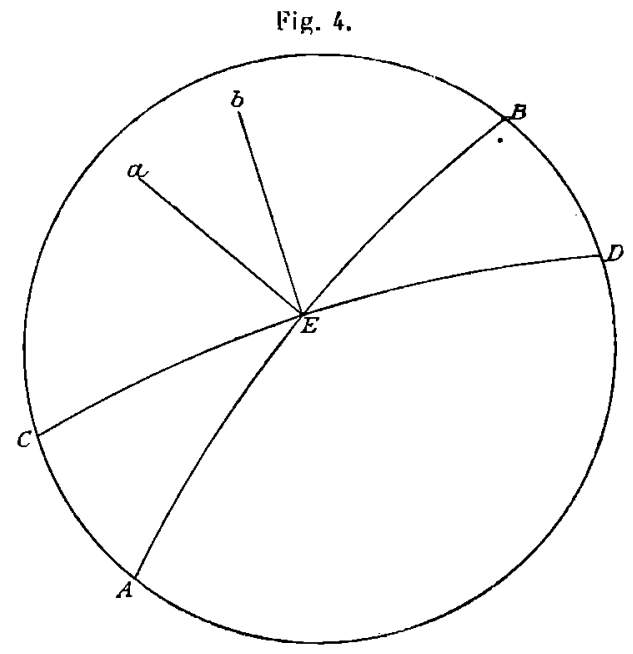

Ziehen wir durch den Punkt $E$ zwei Normalen $E a$ und $E b$ (zu $A B$ und zu $C D)$, so ist der Winkel $a E b$ der gesuchte.

Es mögen nun Aufgaben anderer Art folgen, und zwar solche, bei welchen die Transformation der Projectionsebene im Vordergrunde steht.

Da gerade die Aufgaben dieser Art zu den häufigsten und gewöhnlichsten bei meinen Untersuchungen gehörten, so suchte ich die einfachste und rascheste Methode sie aufzulösen.

Zu diesem Zwecke habe ich auf durchsichtigem Papiere Projeclionen von zweierlei Art drucken lassen, welche aus den Figg. 5 und 6 sogleich verständlich sind.

Fig. 5 .

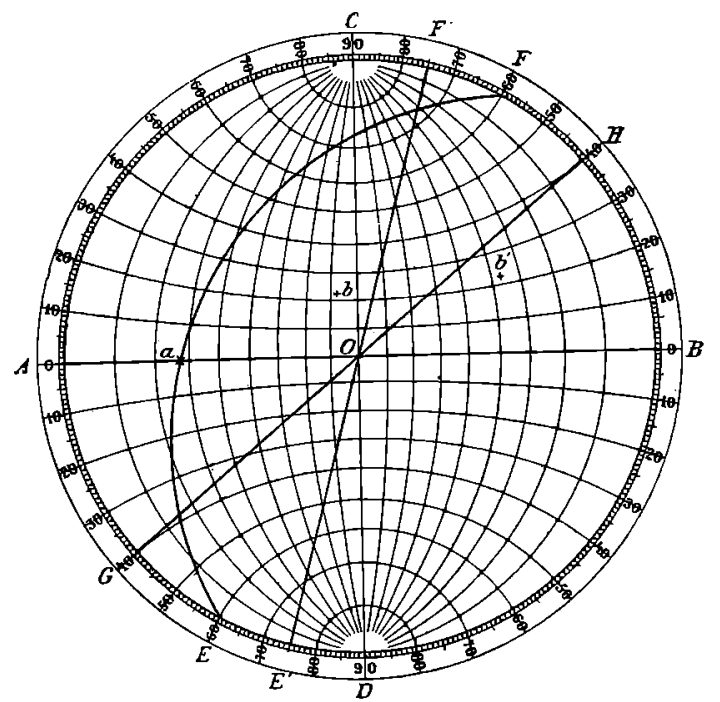

Der grösseren Genauigkeit willen gab ich diesen Projectionen Durchmesser von $20 \mathrm{~cm}$. 
Vierte Aufgabe. Die Projectionsebene ist derart zu transformiren, dass der Pol $a$ die Lage $O$ des Centrums annimmt. Gesucht die neue Lage des Poles $b^{*}$ ) (Fig. 5 auf vor. Seite).

In diesem Falle ist die Transformation so aufzufassen, als ob das System mit den Punkten $a$ und $b$ um den zur Gerade $a O$ senkrechten Durchmesser $C D$ als Axe gedreht wird.

Man legt dabei die auf dem durchsichtigen Papiere gedruckte Projection Fig. 5 auf die gegebene derart, dass die (gleichen) Projectionskreise zusammenfallen und zugleich der Diameter $\boldsymbol{A} B$ mit der Geraden $a O$.

Dann liest man auf der Projection unmittelbar ab, um wieviel Grade das System gedreht ist. Das Ablesen geschieht fast bis auf $\frac{1}{2}$ Grad genau, und dies genügt vollständig für die von mir ausgeführten optischen Untersuchungen der Feldspathe.

Weiss man jetzt diesen Winkel, so ist es leicht, auch die neue Lage $b^{\prime}$ des Punkes $b$ ebenso wie die jedes anderen aufzufinden, indem man die Richtung des durch $b$ hindurchgehenden Kleinkreises der Projection in Gedanken verfolgt und die gefundene Grösse des Winkels in dieser Richtung aufträgt.

Fünfte Aufgabe. Gegeben ein Pol $a$ und ein durch denselben hindurchgehender Zonenkreis $E F$. Die Projection so zu transformiren, dass $a$

Fig. 6.

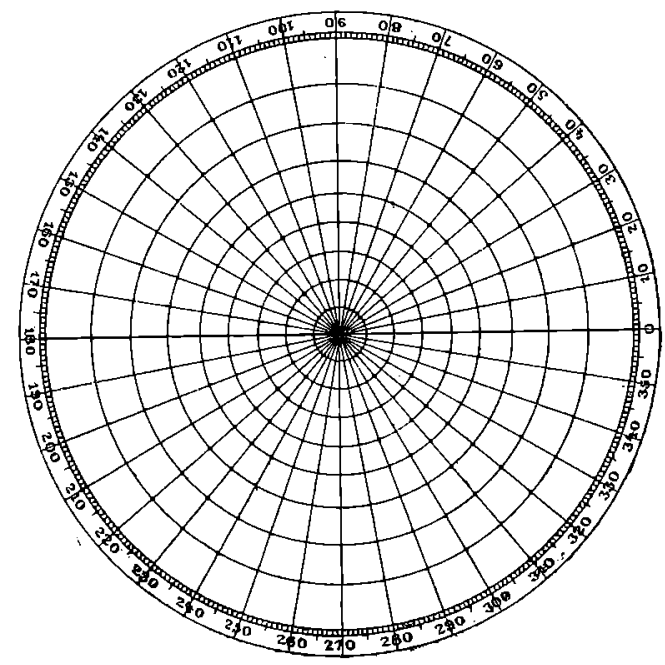

die Lage des Centrums $O$ annimmt, und der Zonenkreis $E F$ die Lage $G H$ (Fig.5).

$Z$ iehe man die Gerade $a O$ und finde, wie in der vorigen Aufgabe, die Lage eines dem Bogen $E a F$ angebörigen Punktes; da finden wir die Gerade $E^{\prime} F^{\prime}$.

Jetzt bleibt nur ubrig die Projection um den Punkt $O$ zu drehen, bis die Gerade $E^{\prime} F^{\prime}$ mit der Geraden $G H$ zusammenfällt, also um den Winkel $\mathrm{F}^{\prime} \mathrm{OH}$, was besonders leicht mit Hülfe der auf durchsichtigem Papiere gedruckten Projection Fig. 6 sich vollziehen lässt.

*) Ueber eine einfache Auflösung dieser Aufgabe durch Construction hat Herr G. Wulff im vorigen Jahre in einer Sitzung der k. mineralog. Ges. zu St. Petersburg vorgetragen. 
Nun ist es leicht, zu der Aufgabe dieser Art in der allgemeinsten Forn uberzugehen, und zwar:

Sechste Aufgabe. Die Transformalion der stereographischen Projection muss derart ausgefuhrt werden, dass dem Pole $a^{\prime}$ die neue Lage $a$, und dem durch $a^{\prime}$ hindurchgehenden Zonenkreise $A^{\prime} B^{\prime}$ die Lage $A B$ zukommt (dabei braucht man nicht die Richtung in dem Zonenkreise ausser Acht zu lassen; diese Richtungen sind durch Pfeile angegeben, Fig. 7).

Ziehen wir durch das Centrum $O$ den zum Zonenkreise $A B$ senkrechten Zonenkreis (Gerade) $0 b$, messen den Winkel $a b$ und finden auf dem Bogen $A^{\prime} B^{\prime}$ den Punkt $b^{\prime}$ (in gehöriger Richtung) dadurch, dass die Grösse des Fig. 7.

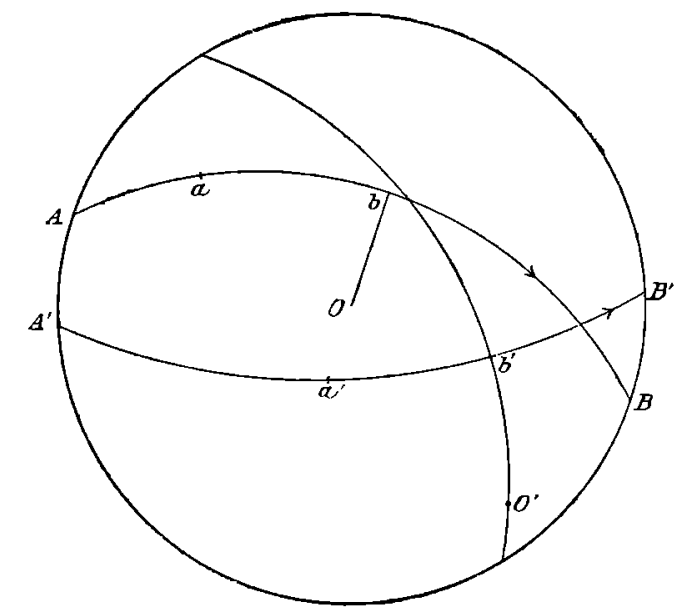
Bogens $a^{\prime} b^{\prime}$ der des Bogens $a b$ gleich ist; endlich ziehen wir durch den Punkt $b^{\prime}$ den zu $A^{\prime} B^{\prime}$ senkrechten Kreisbogen $b^{\prime} O^{\prime}$ und finden den Punkt $O^{\prime}$ dadurch, dass $b^{\prime} O^{\prime}$ gleich $b O$ ist.

Dem Punkte $O^{\prime}$ entspricht jetzt das Centrum und dem Kreisbogen $b^{\prime} O^{\prime}$ der $b o$; die Aufgabe ist also auf die vorige zurückgefubrt worden.

Zum Schluss möchte ich bemerken, dass die einfache Lösung der Aufgabe der Transformation auch die einfachere Auflösung mancher anderer Aufgaben zur Folge hat.

Sollen z. B. die Winkel zwischen den Tracen der Flächen $b, c, d$.. auf der Fläche $a$ aufgefunden werden, so haben wir die Transformation so vorzunehmen, dass der Pol $a$ in die Lage des Projectionscentrums übergefuhrt wird, und finden dann die neuen Lagen von $b, c, d$. Bezeichnen wir die neuen Lagen dieser Pole entsprechend durch $a^{\prime}, b^{\prime}, c^{\prime}, d^{\prime}$. , so sind die Winkel zwischen den Geraden $a^{\prime} b^{\prime}, a^{\prime} c^{\prime}, a^{\prime} d^{\prime} \ldots$ die gesuchten.

Bedeuten die Pole $e^{\prime}, f^{\prime}$ die der optischen Axen, so ergeben sich auch die Halbirenden zwischen den Geraden $a^{\prime} e^{\prime}$ und $a^{\prime} f^{\prime}$, und dies sind die Auslöschungsrichtungen auf der Fläche $a^{\prime}$ resp. $a$. 